\title{
Theory of Glass Transition of Sodium Disilicate Glass
}

\section{-Electronic State between Two Nonbridging Oxygens-}

\author{
By \\ Kazuo KATSUKI and Kozi AOKI \\ (Research Laboratory, Nippon Sheet Glass, Co., Ltd., Itami)
}

\section{Introduction}

In the preceding paper ${ }^{1}$, on the assumption that a sodium disilicate glass is composed of a great number of discrete molecules, its temperature dependence of volume has been calculated thermostatistically on the basis of an assumed potential between oxygens belonging to one discrete ion and another. It is assumed in form that it rises abruptly at a slightly higher point than the minimum point when compared to the Lennard-Jones' type potential. This point is noted to coincide with a critical point when the sodium atoms interact equally with the nonbridging oxygens surrounding these atoms below this point and interact mainly with one of them above this point.

In this paper, this potential curve is investigated by making use of a simple self-consistent field theory $(\mathrm{SCF})^{2)}$. Such calculation as to make clear an electronic state in glass are not available. Because of irregular location of atoms constituting it, except the tetragonal form of $\mathrm{SiO}_{4}$, quantum mechanical treatment of electronic state is very complex for the glass system. Nevertheless, analysis of such a large system can be seen in the biophysical field $^{3)}$. Some atoms, for instance, hydrogen, oxygen and nitrogen atoms, that play an important role as an origin of interaction between biopolymer molecules, are taken off from other atoms in the molecules and only these atoms are taken into account in so far as they interact with each other as $\pi$-electron system. In a similar consideration, but not $\pi$-electron system, we apply SCF procedure to a system which is composed of two nonbridging oxygens neighbouring each other and two sodium atoms interacting with these oxygens. The effect of these atoms on other atoms, even those which belong to the same molecule that any of the four atoms belongs to, is ignored. By analysis of this isolated system, however, we cannot obtain the above-mentioned true potential. That is, a relative displacement of the nonbridging oxygens in the system, amounting to the displacement between the two discrete molecules which bind each of these oxygens respectively, should induce some displacement of the two sodium atoms intermedia- ting the nonbridging oxygens in order that the sodium atoms take on a configuration of the minimum energy of the system. Then each sodium atom causes some variation of its interaction energy with the atoms in the discrete molecules, but that is not taken into account except in the case of the energy with respect to the four atoms. This variation of energy should constitute a part of the interaction energy between the nonbridging oxygens. So as to obviate this complex situation, we introduce an assumption later.

The more atoms are contained in the system, the greater number of molecular integrals have to be carried out for the SCF procedure. And a molecular integral connot be performed analytically if it is formed by coordinates of three-center or four-center atoms. Thus we calculate the molecular integral of poly-center approximately by a method of gaussian expansion of atomic orbital which has been developed and coded for a computer by Huzinaga et $\mathrm{al}^{4)}$.

Now, we turn to a mention of a few assumptions to develop the investigation.

(A) To take account of the model constructed by only the four atoms, we assume a state of interaction between the nonbridging oxygen and silicate atom as follows:

Each nonbridging oxygen atom has tetragonal hybrid orbitals of $s p^{3}$, one of which joins to conform a bond orbital with atomic orbitals on the silicate atoms. Other orbitals on that oxygen atoms do not conform an interacting orbital with any orbitals on the silicate atom at all. We assume that an electron belonging to the oxygen is constrained in that interacting orbital for the $\mathrm{Si}-\mathrm{O}$ bond, and that its effect on the system is ignored.

Consequently the system is composed of 36 electrons and of 20 orbitals.

(B) The field of $3 s$-electron belonging to the sodium atom extends widely compared with the field of other electrons on the same atom, which are pair electron states in closed atomic orbitals. Therefore we assume that only the former moves in the field of the system. The latter are constrained to the ions of sodium atoms, thus their 
effect on the field of system is regarded as that of core electrons.

Similarly, we regard $1 s$-electrons belonging to the oxygen atoms as core electrons.

(C) We assume that the four atoms take positions on a plane and form a configuration of the group symmetry $C_{2 h}$. This suggests that the axis of radial symmetry of the hybrid orbital on the nonbridging oxygen extending to the silicate atom coincides with the line from the oxygen to the silicate atom.

(D) By investigation of the four atoms' model, it is impossible to obtain full information of the interaction between the nonbridging oxygens each belonging to one discrete ion and another. Hence we introduce the following assumption:

If we were able to represent an interaction energy $E^{\prime}$ separately coming from the interaction among the four atoms and their interaction with other atoms in the two discrete ions being in bond with the two nonbridging oxygens in the four atoms respectively, such as an energy between each sodium atom and each nonbridging oxygen numbered with the same subscript (The two nonbridging oxygens are given subscripts 1 and 2 respectively, and the two sodium atoms are also numbered to correspond, with their near oxygens

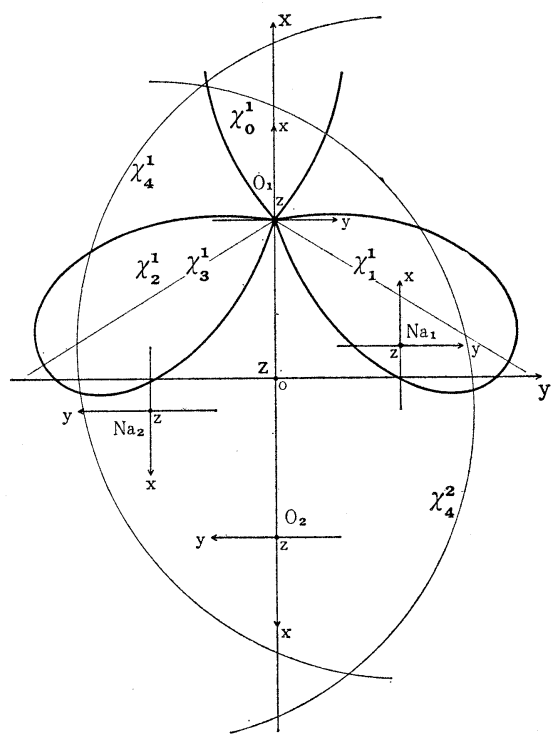

Fig. 1. Configuration of the four atoms. The atomic orbitals on the nonbridging oxygens $\mathrm{O}_{i}(i=1$, 2) form the orthogonal hybrid orbitals of $s p^{8}$, in which $\chi_{0}{ }^{i}$ extending to the silicate atoms and $\chi_{1}{ }^{i}$ are on the plane formed by the four atoms, $\chi_{2}^{i}$ and $\chi_{3}{ }^{i}$ are in symmetrical location to each other with respect to the plane. The orbital $\chi_{4}{ }^{i}$ represent the $3 s$-orbitals on the sodium atoms. The $1 s$-orbitals on the oxygens and the $1 s^{-}, 2 s^{-}$and $2 p$-orbitals on the sodium atoms are regarded as the core orbitals. as shown in Fig. 1), say $V^{\prime}\left(\mathrm{Na}_{i}-\mathrm{O}_{i}\right), i=1,2$, between each sodium atom and the other atoms which constitute one of the discrete ions being bonded with the oxygen having the same subscript, say $V^{\prime}\left(\mathrm{Na}_{i}-D_{i}\right), i=1,2$, and between the nonbridging oxygens with the energy caused by the two sodium atoms interacting on these oxygens, say $V$, therefore the energy $E^{\prime}$ is shown by

$$
\begin{aligned}
E^{\prime}= & V^{\prime}\left(\mathrm{Na}_{1}-D_{1}\right)+V^{\prime}\left(\mathrm{Na}_{2}-D_{2}\right) \\
& +V^{\prime}\left(\mathrm{Na}_{1}-\mathrm{O}_{1}\right)+V^{\prime}\left(\mathrm{Na}_{2}-\mathrm{O}_{2}\right)+V+V_{0}
\end{aligned}
$$

In configuration of the group symmetry $C_{2 h}$, it is rewritten as

$$
E^{\prime}=2 V^{\prime}(\mathrm{Na}-D)+2 V^{\prime}(\mathrm{Na}-\mathrm{O})+V+V_{0}
$$

where $V_{0}$ is independent energy of relative positions of the nonbridging oxygens. In infinite separation between the discrete ions, it becomes

$$
E_{\infty}^{\prime}=2 V^{\prime}(\mathrm{Na}-D)+2 V^{\prime}(\mathrm{Na}-\mathrm{O})+V_{0}
$$

Now, we assume that the sum of the two energies, that is $V^{\prime}(\mathrm{Na}-D)+V^{\prime}(\mathrm{Na}-\mathrm{O})$, does not depend on translational motion of the discrete ions and is nearly constant. For instance, the following phenomenon is presumed to occur: Certain displacement of the sodium atom caused by translational motion of the discrete ions so as to assume such positions as would give rise to the minimum energy of the system lowers (raises) the energy $V^{\prime}(\mathrm{Na}-\mathrm{O})$ between the nonbridging oxygen and the sodium atom, but on the other hand, raises (lowers) the energy $V^{\prime}(\mathrm{Na}-D)$ between that sodium atom and other nonbridging oxygens which are in a bonded state with the same discrete ion as the nonbridging oxygen included in the potential $V^{\prime}(\mathrm{Na}-\mathrm{O})$ is in bonded state with. Thus the interaction energy between the nondridging oxygens intermediated by the two sodium atoms can be denoted by

$$
\begin{aligned}
E^{\prime}-E_{\infty}^{\prime}= & V=\left(E^{\prime}-V_{0}-2 V^{\prime}(\mathrm{Na}-D)\right) \\
& -2 V^{\prime}(\mathrm{Na}-\mathrm{O})
\end{aligned}
$$

We replace $E^{\prime}-V_{0}-2 V^{\prime}(\mathrm{Na}-D)$ by the energy of system $E$ which will be calculated, and the last term by the energy $2 \mathrm{~V}(\mathrm{Na}-\mathrm{O})$, which is obtained in the infinite separation between the nonbridging oxygens keeping the same relative positions between each sodium atom and each nonbridging oxygen as in the finite separation. Of course, we choose the configuration of the lower energy from two configurations which can be formed in the above manner.

Therefore we shall calculate $E$ and $V(\mathrm{Na}-\mathrm{O})$, and investigate the feature of $V$ which has been assumed as the effective potential function in the preceding paper.

\section{SCF procedure}

If the purpose of following SCF procedure is to obtain the accurate interaction energy between the nonbridging oxygen atoms, it is an important 
problem which type of atomic orbitals is used to calculate the best energy state of the system. Nevertheless, our aim is to consider the feature of interaction potential curve, which plays a characteristic role in causing glass transition, represented by a function of the distance between the nonbridging oxygens. Thus we adopt slater type's atomic orbitals.

We have assumed the configuration of the four atoms (Fig. 1) to be the group symmetry $C_{2 h}$, and divided them into the two groups. We denote the atomic orbitals by $f_{j}{ }^{i}, i=1,2, j=1 \sim 5$, $11 \sim 16$ (The superscript $i$ means the above mentioned group.) defined by the order of elements in matrix which is shown by

$$
\begin{aligned}
& {\left[f_{1}{ }^{i}, f_{2}{ }^{i}, \cdots, f_{5}{ }^{i}, f_{11}{ }^{i}, \cdots, f_{16}{ }^{i}\right] \cdots \cdots \cdots \cdots(1)} \\
& \equiv\left[2 s\left(\mathrm{O}_{i}\right), x\left(\mathrm{O}_{i}\right), y\left(\mathrm{O}_{i}\right), z\left(\mathrm{O}_{i}\right), 3 s\left(\mathrm{Na}_{i}\right)\right. \text {, } \\
& 1 s\left(\mathrm{O}_{i}\right), 1 s\left(\mathrm{Na}_{i}\right), 2 s\left(\mathrm{Na}_{i}\right), x\left(\mathrm{Na}_{i}\right) \text {, } \\
& \left.y\left(\mathrm{Na}_{i}\right), z\left(\mathrm{Na}_{i}\right)\right], i=1,2
\end{aligned}
$$

where the $2 s$-orbitals on the oxygen atoms, and the $2 s$ - and $3 s$-orbitals on the sodium atoms have been used as orthogonal functions mutually to each other on the same atoms formed in the usual way from original $s$-functions. The hybrid orbitals $\chi_{j}{ }^{i}, i=1,2 j=0 \sim 3$ on the oxygen atoms which are assumed in (A) of section 1 are formed by a matrix $\boldsymbol{\beta}^{\prime}$ from the orbitals $f_{j}{ }^{i}, i=1,2, j=1 \sim 4$. These are defined as the elements of martrix

$$
\left[\chi_{0}{ }^{i}, \cdots, \chi_{3}{ }^{i}\right]=\left[f_{1}{ }^{i}, \cdots, f_{4}{ }^{i}\right] \boldsymbol{\beta}^{\prime} i=1,2 \cdots(2)
$$

In the hybrid orbitals, as $\chi_{0}{ }^{i}, i=1,2$ contribute only to the interaction of the oxygens with the silicate atoms, we omit them from the basic orbitals that are used to conform the interaction orbitals in the system. For that, we newly define $\boldsymbol{\chi}, \boldsymbol{\beta}$ and $\boldsymbol{f}$ matrices by

$$
\begin{aligned}
& \boldsymbol{\chi}=\left[\chi_{1}, \cdots, \chi_{8}\right] \\
& \boldsymbol{f}=\left[f_{1}{ }^{1}, \cdots, f_{5}{ }^{1}, f_{1}{ }^{2}, \cdots, f_{5}{ }^{2}\right]
\end{aligned}
$$

and

$$
\beta=\left(\begin{array}{ccclc}
1 / 2 & 1 / 2 & 1 / 2 & 0 & \\
-1 / 2 \sqrt{3} & -1 / 2 \sqrt{3} & -1 / 2 \sqrt{3} & 0 & \\
\sqrt{2} / \sqrt{3} & -1 / \sqrt{6} & -1 / \sqrt{6} & 0 & \\
0 & 1 / \sqrt{2} & -1 / \sqrt{2} & 0 & \\
0 & 0 & 0 & 1 & \\
& & & & 1 / 2 \\
& & & & -1 / 2 \sqrt{3} \\
& 0 & & & \sqrt{2} / \sqrt{3} \\
& & & & 0 \\
& & & & 0
\end{array}\right.
$$

The elements on the left-hand side of (2) have been replaced by $\chi_{i}$ except $\chi_{4}$ and $\chi_{8}$ according to the following relation,

$$
\chi_{q}{ }^{p}=\chi_{4(p-1)+q} \cdots \cdots \cdots \cdots \cdots \cdots \cdots \cdots \cdots \cdots \cdots \cdots \cdots \cdots \cdots \cdots \cdots \cdots \cdots \cdots
$$

\begin{tabular}{|c|c|}
\hline Symmetrical spacies & Orbitals \\
\hline $\mathrm{Ag}$ & $\left\{\begin{array}{l}\sigma_{1}=\alpha\left(\chi_{1}^{1}+\chi_{1}{ }^{2}\right) \\
\sigma_{2}=\alpha^{2}\left(\chi_{2}{ }^{1}+\chi_{2}{ }^{2}+\chi_{3}{ }^{1}+\chi_{3}{ }^{2}\right) \\
\sigma_{3}=\alpha\left(\chi_{4}{ }^{1}+\chi_{4}{ }^{2}\right)\end{array}\right.$ \\
\hline $\mathrm{Au}$ & 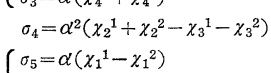 \\
\hline $\mathrm{Bu}$ & $\left\{\begin{array}{l}\sigma_{6}=\alpha^{2}\left(\chi_{2}{ }^{1}-\chi_{2}{ }^{2}+\chi_{3}{ }^{1}-\chi_{3}{ }^{2}\right) \\
\sigma_{7}=\alpha\left(\chi_{4}{ }^{1}-\chi_{4}{ }^{2}\right)\end{array}\right.$ \\
\hline $\mathrm{Bg}$ & $\sigma_{8}=\alpha^{2}\left(\chi_{2}{ }^{1}-\chi_{2}{ }^{2}-\chi_{3}{ }^{1}+\chi_{3}{ }^{2}\right)$ \\
\hline
\end{tabular}

Thus the set of the hybrid orbitals is formed from the atomic orbitals by

$$
\boldsymbol{\chi}=\boldsymbol{f} \boldsymbol{\beta}
$$

The interaction orbitals have to belong in sets to irreducible representation of symmetry group $C_{2 h}$
Table 1. Symmetrical orbitals $(\alpha=1 / \sqrt{2})$

mentioned before. To get this representation, it is convenient to transform $\chi$ into group of symmetrical orbitals $\boldsymbol{\sigma}$. If we denote transformation matrix by $\boldsymbol{U}$, these orbitals are written as

$$
\boldsymbol{\sigma}=\boldsymbol{\chi} \boldsymbol{U}
$$

The resultant symmetrical orbitals with the irreducible representation of the group $C_{2 h}$ are given in Table 1. By taking linear combinations of these symmetrical orbitals, the interaction orbitals $\Phi$, of which each orbital belongs to one of the group $C_{2 h}$, are obtained and they are written by matrix representation as

$\boldsymbol{\Phi}=\boldsymbol{\sigma} \boldsymbol{b}$

where $\boldsymbol{b}$ is the unknown matrix to be determined and is composed of $3 \times 3,1 \times 1,3 \times 3$ and $1 \times 1$ small matrices referring to Table 1 .

Now, we construct an effective Hamiltonian Operator to determine the best interaction orbitals $\Phi$. In this process it is doubtful whether the $2 p$-orbitals on the two sodium atoms are assumed to play a role of the core orbitals in the system, but we continue the procedure regarding this assumption as involving the same degree of approximation in ignoring the effect of the silicate atoms on the system.

We write one electron operator as

$$
-\frac{1}{2} \nabla_{i}^{2}+\Sigma \frac{Z_{N}}{\left|\boldsymbol{r}_{i}-\boldsymbol{R}_{N}\right|}
$$

where $\Sigma$ before
0

$\left.\begin{array}{cc} & \\ & \\ 1 / 2 & 0 \\ -1 / 2 \sqrt{3} & 0 \\ -1 / \sqrt{6} & 0 \\ -1 / \sqrt{2} & 0 \\ 0 & 1\end{array}\right] \cdots(5)$

the nuclear attraction energy operator indicates the sum of $N$ with respect to the coordinates $\boldsymbol{R}_{1}$, $\boldsymbol{R}_{2}, \boldsymbol{R}_{3}$ and $\boldsymbol{R}_{4}$, and nuclear charges $Z_{1}, Z_{2}, Z_{3}$ and $Z_{4}$ standing for the four atoms $\mathrm{O}_{1}, \mathrm{Na}_{1}, \mathrm{O}_{2}$ and $\mathrm{Na}_{2}$ respectively. There is another one electron operator which comes from the core electrons.

Therefore the Hamiltonian matrix $\boldsymbol{H}$ is written by $\boldsymbol{I}, \boldsymbol{L}$ and the supermatrix, say $(2 \boldsymbol{J}(t)-\boldsymbol{K}(t))$, of the 8 interaction orbitals in the system, whose elements are shown by

$$
\boldsymbol{I}_{p, q}=\left\langle\sigma_{p}\left|-\frac{1}{2} \nabla^{2}+\Sigma \frac{Z_{N}}{\left|\boldsymbol{r}_{i}-\boldsymbol{R}_{N}\right|}\right| \sigma_{q}\right\rangle
$$




$$
\begin{aligned}
\boldsymbol{L}_{p, q} & \equiv \sum_{\substack{m=1,2 \\
n=11 \sim 16}} \boldsymbol{L}_{p, q}(m, n) \\
& =\sum_{\substack{m=1,2 \\
n=11 \sim 16}}\left[2\left\langle f_{n}{ }^{m}, \sigma_{p} \mid f_{n}{ }^{m}, \sigma_{q}\right\rangle\right. \\
& \left.-\left\langle f_{n}^{m}, \sigma_{q} \mid \sigma_{p}, f_{n}{ }^{m}\right\rangle\right] \quad \ldots \ldots \ldots . . .
\end{aligned}
$$

and

$$
\begin{aligned}
& (2 \boldsymbol{J}(t)-\boldsymbol{K}(t))_{p q} \\
& \quad=2\left\langle\phi_{t}, \sigma_{p} \mid \phi_{t}, \sigma_{q}\right\rangle-\left\langle\phi_{t}, \sigma_{p} \mid \sigma_{q}, \phi_{t}\right\rangle
\end{aligned}
$$

where

$$
\begin{aligned}
& \left\langle\alpha_{1}|\emptyset| \alpha_{2}\right\rangle=\int \alpha_{1}(\boldsymbol{r}) \bigvee \alpha_{2}(\boldsymbol{r}) d \boldsymbol{r}, \\
& \left\langle\alpha_{1}, \alpha_{2} \mid \alpha_{3}, \alpha_{4}\right\rangle=\int \frac{\alpha_{1}\left(\boldsymbol{r}_{i}\right) \alpha_{2}\left(\boldsymbol{r}_{j}\right) \alpha_{3}\left(\boldsymbol{r}_{i}\right) \alpha_{4}\left(\boldsymbol{r}_{j}\right)}{\left|\boldsymbol{r}_{i j}\right|} . \\
& \cdot d \boldsymbol{r}_{i} d \boldsymbol{r}_{j}
\end{aligned}
$$

By making use of these matrices' elements, we obtain the secular equation to determine the best interaction orbitals, which is

$$
\boldsymbol{H} \boldsymbol{b}=\boldsymbol{\varepsilon} \boldsymbol{S} \boldsymbol{b}
$$

where $\boldsymbol{S}$ indicates overlap matrix, which element is written by

$$
\boldsymbol{S}_{p, q}=\left\langle\sigma_{p} \mid \sigma_{q}\right\rangle
$$

and $\boldsymbol{H}$ is written as

$$
\boldsymbol{H}=\boldsymbol{I}+\boldsymbol{L}+\sum_{t=1}^{6}(2 \boldsymbol{J}(t)-\boldsymbol{K}(t))
$$

By solving the secular equation, we can obtain 8 interaction orbitals and their corresponding orbital energies, but only the 6 orbitals $\phi_{1}, \phi_{2}, \cdots, \phi_{6}$ that are taken from the orbitals giving rise to the lower orbital energies of the calculated 8 orbitals are occupied orbitals in the system, because the system is composed of the 12 valence electrons, and each orbital is a closed one. (The two electons belong to the $3 s$-orbitals on the two sodium atoms and the remainder divided equally on the two oxygen atoms belong to the hybrid orbitals.) Consequently a part of the potential energy $E$ which comes from the valence electron interaction is obtained by

$$
\begin{aligned}
E_{V}= & 2 \sum_{i=1}^{6} \varepsilon_{i}-\sum_{i, j}^{6} \boldsymbol{b}_{i}+(2 \boldsymbol{J}(j)-\boldsymbol{K}(j)) \boldsymbol{b}_{i} \\
& -\sum_{i}^{6} \boldsymbol{b}_{i}{ }^{+} \boldsymbol{L} \boldsymbol{b}_{i}+\frac{1}{2} \Sigma^{\prime} \frac{Z_{N} Z_{N^{\prime}}}{\left|\boldsymbol{R}_{N}-\boldsymbol{R}_{N^{\prime}}\right|}
\end{aligned}
$$

where the last term is, of course, bare nuclear repulsion energy and $\Sigma^{\prime}$ means the sum of $N$ and $N^{\prime}$ except $N=N^{\prime}$. The core orbital energies $\varepsilon_{c}(m, n)$ are calculated by substituting $f_{n}{ }^{m}$ for $\sigma_{p}$ and $\sigma_{q}$ in $\boldsymbol{I}_{p, q}$ and $f_{n_{1}}^{m_{1}}$ for $\phi_{t}$ and $f_{n_{2}}^{m_{2}}$ for $\sigma_{p}, \sigma_{q}$ in $(2 \boldsymbol{J}(t)-\boldsymbol{K}(t))_{p, q}$ for which we denote $\boldsymbol{I}_{m, n}^{c}$ and ( $\left.2 \boldsymbol{J}\left(m_{1}, n_{1}\right)-\boldsymbol{K}\left(m_{1}, n_{1}\right)\right)_{m_{2}, n_{2}}^{c}$ respectively. As a result, the core orbital energies are obtained by

$$
\begin{aligned}
& \varepsilon_{c}(m, n)=\boldsymbol{I}_{m, n}^{c}+\sum_{\substack{m_{1}=1,2 \\
n_{1}=11 \sim 16}}\left(2 \boldsymbol{J}\left(m_{1}, \mathrm{n}_{1}\right)\right. \\
& \left.-\boldsymbol{K}\left(m_{1}, n_{1}\right)\right)_{m, n}^{c}+\sum_{i}^{6} \boldsymbol{b}^{+}{ }_{i} \boldsymbol{L}(m, n) \boldsymbol{b}_{i}, m=1,2 \text {, } \\
& n=11 \sim 16
\end{aligned}
$$

where $\varepsilon_{c}(m, n)$ is independent of $m$ because of the symmetrical configuration. energy $E$ is shown by

$$
E=E_{V}+E_{c}
$$

where

$$
\begin{aligned}
E_{c}= & 2\left[2 \sum_{n=11}^{16} \varepsilon_{c}(1, n)-\sum_{n=11}^{16} \sum_{\substack{m_{1}=1,2 \\
n_{1}=11 \sim 16}}\left(2 \boldsymbol{J}\left(m_{1}, n_{1}\right)\right.\right. \\
& \left.\left.-\boldsymbol{K}\left(m_{1}, n_{1}\right)\right)_{1, n}^{c}-\sum_{n=11}^{16} \sum_{i}^{6} \boldsymbol{b}_{i}^{+} \boldsymbol{L}(1, n) \boldsymbol{b}_{i}\right]
\end{aligned}
$$

In the infinite separation between the two nonbridging oxygens, 4 interaction orbitals $\psi_{i}, i=1$, $\cdots, 4$, in which 3 orbitals are occupied orbitals, are formed by linear combinations of $\chi_{p}, p=1$, .., 4 which are formed from atomic orbitals numbered with the same subscript. Applying the same method as used in the preceding calculation to this case, we obtain $V(\mathrm{Na}-\mathrm{O})$.

\section{Numerical calculation and discussion}

In order to draw the potential curve between the nonbridging oxygens in the system, location of the two sodium atoms that minimizes the total energy as a functional of the O-O distance must be determined. To do that, first of all, the four atoms are fixed at arbitrary positions of which configuration forms the group symmetry $C_{2 h}$, and the total energy with respect to this configuration is calculated. Next, only the sodium atoms are transferred to other positions keeping the group symmetry $C_{2 h}$, and then the energy corresponding to this configuration is obtained. We continue this process until the minimum energy is found on the condition of the fixed distance between the nonbridging oxygens. The next step is to change the distance between the nonbridging oxygens and to repeat the above process to determine the positions of sodium atoms giving rise to the minimum energy on this $\mathrm{O}-\mathrm{O}$ distance. Finally, we can draw the potential curve of the minimum energy represented by the corresponding $\mathrm{O}$ $\mathrm{O}$ distance.

If we apply the SCF procedure to the electronic system described in the preceding section according to the above mentioned process, it is very time-consuming. (For instance, it takes about six and a half hours for molecular integrals per configuration, and the same number of molecular integrals have to be carried out for every other configuration.) Therefore we simplify the system by neglecting the core orbitals in order to determine the positions of sodium atoms giving rise to the minimum energy on each fixed $\mathrm{O}-\mathrm{O}$ distance. That is; the system is composed of 12 electrons and 8 orbitals, the nuclear charges have to be replaced by 6 and 1 for $Z_{1}$ and $Z_{2}$ respectively. In consequence, the positions of sodium atoms with the above mentioned meaning are determined as shown in Table 2 .

One can see from Table 2 that the two sodium 
Table 2. Positions $(X, Y, Z)$ of Sodium Atom that give rise to the minimum energy in each $\mathrm{O}-\mathrm{O}$ distance (atomic unit).

\begin{tabular}{cc}
\hline Oxygen-Oxygen distances & Positions of sodium atom \\
\hline 2.50 & $(0,1.770,0)$ \\
2.60 & $(0,1.710,0)$ \\
2.78 & $(0,1.640,0)$ \\
2.84 & $(0,1.625,0)$ \\
2.90 & $(0,1.620,0)$ \\
3.02 & $(0,1.600,0)$ \\
3.08 & $(0,1.580,0)$ \\
3.14 & $(0.150,1.575,0)$ \\
3.20 & $(0.200,1.575,0)$ \\
3.30 & $(0.330,1.550,0)$ \\
\hline
\end{tabular}

atoms move on a line keeping symmetrical positions with respect to the origin below a certain distance between the two nonbridging oxygens which is perpendicular to the line connecting the oxygens, crossing it at the middle point between them, and that each sodium atom changes its direction of movement from that line to the one oxygen and the other respectively above that certain distance in order to take on the configuration giving rise to the minimum energy. This critical distance has been denoted as $a_{2}$ in the preceding paper which played an important role in the origin of glass transition. By the variation of sodium atoms' positions one may presume that each sodium atom interacts equally with the two oxygens below this critical distance.

Let us examine the energy surfaces of the two sodium atoms at every fixed $\mathrm{O}-\mathrm{O}$ distance, which are formed by taking the $Z$ axis of the enrgy value as functional of the positions $X$ and $Y$ of sodium atoms on the similar coordinate to Fig. 1. Their minimum points are on the $Y-Z$ plane in the shorter of the distances and a curve, which is determined as the cross between the energy surface and the $Y-Z$ plane, rises to both sides from the two minimum points along the $Y$ axis, but forms the peak on the origin which is the middle point of the two minimum points. On the other hand, another curve, which is determined as the cross between the energy surface and a plane parallel to the $X-Z$ plane through one of the lowest points, simply increases from the minimum point. The $\mathrm{O}-\mathrm{O}$ distance becomes shorter, the top of hill higher because the minimum points of the energy surface are far from each other. Contrarily, it becomes longer in the range when the minimum points are on the $Y-Z$ plane, the bottom of the energy surface becoming more and more flat. Finally, the minimum points of the energy surface go out from the $Y-Z$ plane and the curve determined in the latter manner also possesses a peak on a point across the $Y-Z$ plane if the two sodium atoms change their positions at the same time so as to interact mainly with the one nonbridging oxygen and the other respectively. (As seen in Fig. 1, the two sodium atoms change their positions to the symmetrical ones with respect to the $Y$ axis, but the resultant state does not change.) Thus the moving space of the sodium atoms increases suddenly above the $a_{2}$ point.

Now, we shall investigate the electronic state of system. For this purpose, we turn to the original model formed by the core and valence orbitals, and on the basis of this model we carry out numerical calculations applying the SCF procedure to the electrons in the four atoms' system which forms the configuration determined by the simplified model. From the result we obtain the following interacting state of the electrons.

In general, each characteristic of the interaction orbitals which are presumed by taking account of the coefficients making linear combinations of the symmetrical orbitals is held independently of the $\mathrm{O}-\mathrm{O}$ distance within our calculation, and there is no bonding orbital common to all the atoms. The orbital $1 a_{g}$ which gives rise to the lowest orbital energy is a bonding orbital with respect to the oxygens, but contributes as an antibonding to interaction between each oxygen and each sodium atom. On the other hand, the orbital $2 a_{g}$ is a main bonding orbital with respect to the sodium atoms, and is nearly nonbonding with respect to the oxygens. Regarding its interaction between the orbitals on the oxygens and sodium atoms, the basic orbitals $\chi_{4}{ }^{1}$ and $\chi_{4}{ }^{2}$ are bonding with $\chi_{1}{ }^{1}$ and $\chi_{1}{ }^{2}$ and antibonding with $\chi_{2}{ }^{1}, \chi_{3}{ }^{1}, \chi_{2}{ }^{2}$ and $\chi_{3}{ }^{2}$. The orbital $3 a_{g}$ acts as an antibonding between the two oxygens. With regard to its sodiumoxygen interaction, $\chi_{4}{ }^{1}$ and $\chi_{4}{ }^{2}$ are bonding with $\chi_{2}{ }^{1}, \chi_{3}{ }^{1}, \chi_{2}{ }^{2}$ and $\chi_{3}{ }^{2}$, and antibonding with $\chi_{1}{ }^{1}$ and $\chi_{1}{ }^{2}$. The orbital $1 b_{u}$ is bonding weakly with respect to the oxygens. Judging from these characteristics of the interaction orbitals, we can see that each sodium atom is connected with the two oxygens by the orbitals which are extending to the sodium atoms from the oxygens. The orbitals $a_{u}$ and $b_{g}$ which are formed from the hybrid orbitals on the two oxygens are perpendicular to the plane made by the four atoms, and the former is bonding with respect to the oxygens and the latter antibonding. Of course, they are nonbonding with respect to the sodium atoms. The orbitals $2 b_{u}$ and $3 b_{u}$ are virtual orbitals. These bonding properties can also be seen by inspecting variations of the orbital energies shown in Table 3. In particular, the orbital $1 b_{u}$ elucidates the phenomenon that the sodium atom mainly interacts on one of the oxygens at the longer of the $\mathrm{O}-\mathrm{O}$ distances and begins to interact equally on one more oxygen at the shorter of the $\mathrm{O}-\mathrm{O}$ distances, so that its interaction energy lowers 
Table 3. Orbital Energies in each O-O Distance (atomic unit).

\begin{tabular}{ccccccccc}
\hline O-O distances & $1 a_{g}$ & $2 a_{g}$ & $3 a_{g}$ & $1 b_{u}$ & $a_{u}$ & $b_{g}$ & $2 b_{u}$ & $3 b_{u}$ \\
\hline 2.50 & -3.1372 & -2.5124 & -1.8987 & -2.5319 & -1.9437 & -1.6783 & 0.2315 & 10.9443 \\
2.60 & -3.1673 & -2.5010 & -1.9094 & -2.5154 & -1.9278 & -1.6878 & 0.0543 & 11.4436 \\
2.78 & -3.1530 & -2.4876 & -1.8988 & -2.4216 & -1.8887 & -1.6913 & -0.1973 & 12.0393 \\
2.84 & -3.1316 & -2.4841 & -1.8895 & -2.3822 & -1.8736 & -1.6894 & -0.2634 & 12.1605 \\
2.90 & -3.0908 & -2.4820 & -1.8752 & -2.3353 & -1.8556 & -1.6847 & -0.3179 & 12.1760 \\
3.02 & -3.0281 & -2.4755 & -1.8503 & -2.2538 & -1.8246 & -1.6768 & -0.4167 & 12.3194 \\
3.08 & -3.0142 & -2.4704 & -1.8411 & -2.2215 & -1.8127 & -1.6747 & -0.4647 & 12.5044 \\
3.14 & -2.9948 & -2.4978 & -1.8313 & -2.2090 & -1.7998 & -1.6731 & -0.5103 & 12.6028 \\
3.20 & -2.9732 & -2.5173 & -1.8201 & -2.2091 & -1.7855 & -1.6700 & -0.5504 & 12.5553 \\
3.30 & -2.9684 & -2.5278 & -1.8046 & -2.2050 & -1.7678 & -1.6668 & -0.6167 & 12.7289 \\
\hline
\end{tabular}

Table 4. Electron Populations in the Basic Orbitals in each O-O Distance $(i=1,2)$.

\begin{tabular}{cccc}
\hline O-O distances & $\chi_{1}{ }^{i}$ & $\chi_{2}{ }^{2}, \chi_{3}{ }^{i}$ & $\chi_{4}{ }^{i}$ \\
\hline 2.50 & 1.6721 & 1.6644 & 0.9991 \\
2.60 & 1.6725 & 1.6642 & 0.9990 \\
2.78 & 1.6733 & 1.6639 & 0.9989 \\
2.84 & 1.6735 & 1.6638 & 0.9989 \\
2.90 & 1.6738 & 1.6637 & 0.9989 \\
3.02 & 1.6742 & 1.6635 & 0.9989 \\
3.08 & 1.6744 & 1.6634 & 0.9989 \\
3.14 & 1.7578 & 1.6216 & 0.9990 \\
3.20 & 1.8084 & 1.5963 & 0.9991 \\
3.30 & 1.8516 & 1.5746 & 0.9992 \\
\hline
\end{tabular}

remarkably from 3.14 of the $\mathrm{O}-\mathrm{O}$ distance. Judging from these properties of orbitals, naturally the electrons belonging to the sodium atoms are not constrained to any atoms, therefore the tendency of ionic bonding does not seem to be present in this system. We show electron populations calculated according to Mulliken's method in Table $4^{5}$. One may see from it that the $3 s$-orbital on the sodium atom keeps nearly one electron in probability the same as in its atomic state, and that electron populations of the orbitals on the oxygens vary from each other as a functional of the $\mathrm{O}-\mathrm{O}$ distance. In the shorter part of the distance between the oxygens, the six orbitals $\chi_{1}{ }^{i}, \chi_{2}{ }^{i}$ and $\chi_{3}{ }^{i},(i=1,2)$, hold electrons nearly equally, but in the longer part of the distance the electron localizes a little in $\chi_{1}{ }^{1}$ and $\chi_{1}{ }^{2}$ which extend to each sodium atom from each oxygen belonging to the same group respectively. Here again one can see the above-mentioned critical phenomenon in the abrupt change of the electron populations in $\chi_{1}{ }^{1}$ and $\chi_{1}{ }^{2}$ between 3.08 and 3.14 of the O-O distance.

We turn our attention now to the infinite separation between the oxygens formed according to the manner mentioned in the preceding section.

A remarkable difference occurs in the case of the interaction state from the state of the finite separation. As shown in Table 5, a large probability of electron in the $3 s$-orbital on the sodium atom transfers to the orbitals on the oxygens, thus the interaction is constituted almost by ionic bonding. On comparing the interactions in the two models,
Table 5. Electron Populations in the Basic Orbitals in the Infinite Separation between the oxygens represented by the corresponding $\mathrm{O}-\mathrm{O}$ distances (refer to Table 2).

\begin{tabular}{cccc}
\hline $\begin{array}{l}\text { Indicators of relative positions of } \\
\text { the oxygen and sodium atom }\end{array}$ & $\chi_{1}{ }^{i}$ & $\chi_{2}{ }^{i}, \chi_{3}{ }^{i}$ & $\chi_{4}{ }^{i}$ \\
\hline 2.50 & 1.9464 & 1.9993 & 0.0550 \\
2.60 & 1.9489 & 1.9963 & 0.0586 \\
2.78 & 1.9431 & 1.9920 & 0.0730 \\
2.84 & 1.9389 & 1.9908 & 0.0794 \\
2.90 & 1.9322 & 1.9901 & 0.0877 \\
3.02 & 1.9200 & 1.9885 & 0.1029 \\
3.08 & 1.9158 & 1.9876 & 0.1090 \\
3.14 & 1.9432 & 1.9862 & 0.0844 \\
3.20 & 1.9550 & 1.9858 & 0.0734 \\
3.30 & 1.9618 & 1.9840 & 0.0702 \\
\hline
\end{tabular}

one can see that the interaction of sodium with the oxygens has the property of strong ionic bonding if these atoms belong to an isolated molecule. On the other hand, of rather covalent bonding in the glass system because the probability of finding the nonbridging oxygens corresponding to the four atoms' model of the finite separation between the oxygens seems to be greater than that of finding the nonbridging oxygens corresponding to the two atoms' model of the infinite separation. The orbitals $\chi_{2}{ }^{i}$ and $\chi_{3}{ }^{i}$ on the oxygen, which are orthogonal to $\chi_{1}{ }^{i}$ respectively, form nearly perfect lone pair orbitals, and that tendency is the stronger at the location of the sodium and oxygen atom represented by the corresponding shorter distance between the oxygens in the finite separation. With regard to bonding properties of the orbitals, the orbital possessing the lowest orbital energy is bonding and its constituent orbital $\chi_{1}{ }^{i}$ receives more electrons from the $3 s$-orbital at the shorter distance between the sodium-oxygen atom. This orbital takes on a characteristic state in energy variation: As presumed from Table 5, other occupied orbital energies vary consistently in accordance with the configuration denoted by the $\mathrm{O}-\mathrm{O}$ distance in the finite separation. However, this orbital energy varies independently of that configurational representation, but varies depending on the oxygen-sodium distance. The orbital of the next orbital energy is bonding with respect to interaction between the $3 s$ and $\chi_{1}{ }^{i}$, but antibonding 


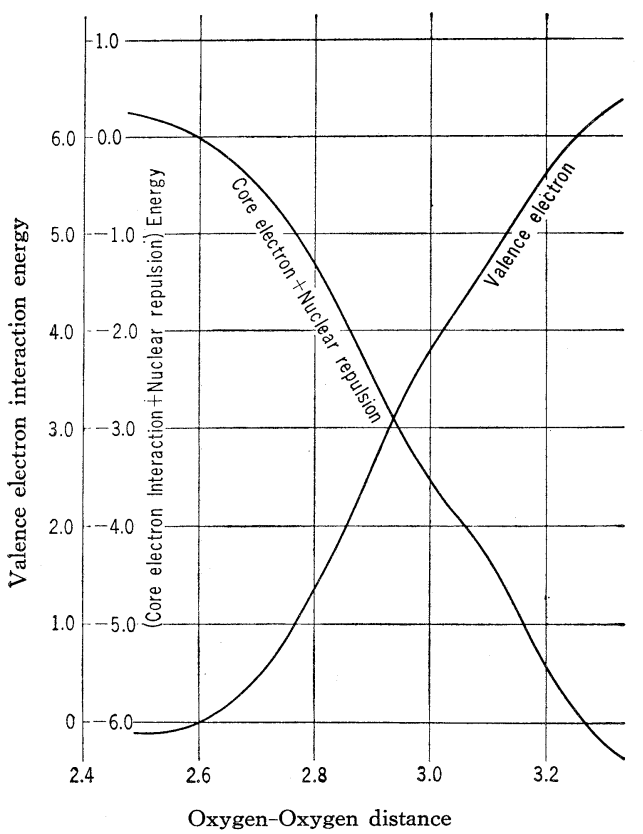

Fig. 2. Valence electron interaction energy and (core electron interaction + nuclear repulsion) energy in the finite separation between the nonbridging oxygens. The values at 2.60 of the $\mathrm{O}-\mathrm{O}$ distance are taken as the base values.

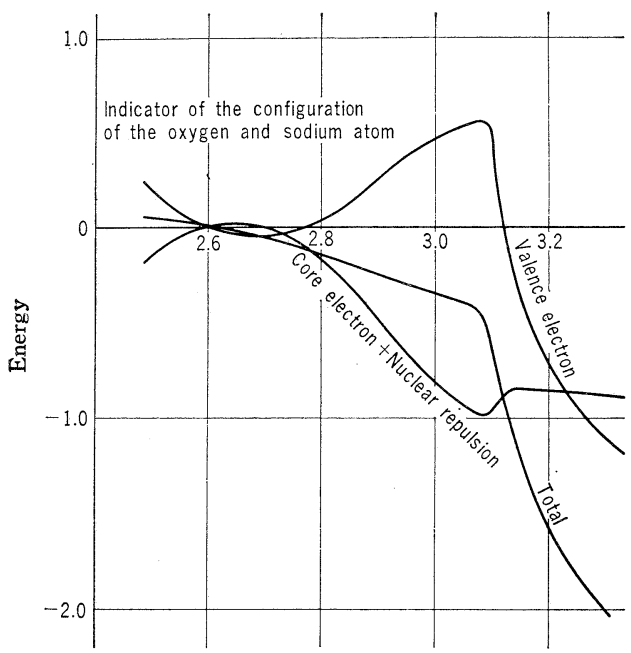

Fig. 3. Interaction energy between the nonbridging oxygen and sodium atom in the infinite separation between the nonbridging oxygens, where the abscissa indicates the configuration of the nonbridging oxygen and sodium numbered with the same subscript as in the finite separation between the oxygens. The values at 2.60 of the indicator are taken as the base values.

between $3 s$ and $\chi_{2}{ }^{i}$ or $\chi_{3}{ }^{i}$. Another occupied orbital formed from $\chi_{2}{ }^{i}$ and $\chi_{3}{ }^{i}$ is nonbonding.

Next, we consider the system energy of both the finite and infinite separation between the oxygens. We show them in Fig. 2, 3 and 4 represen-

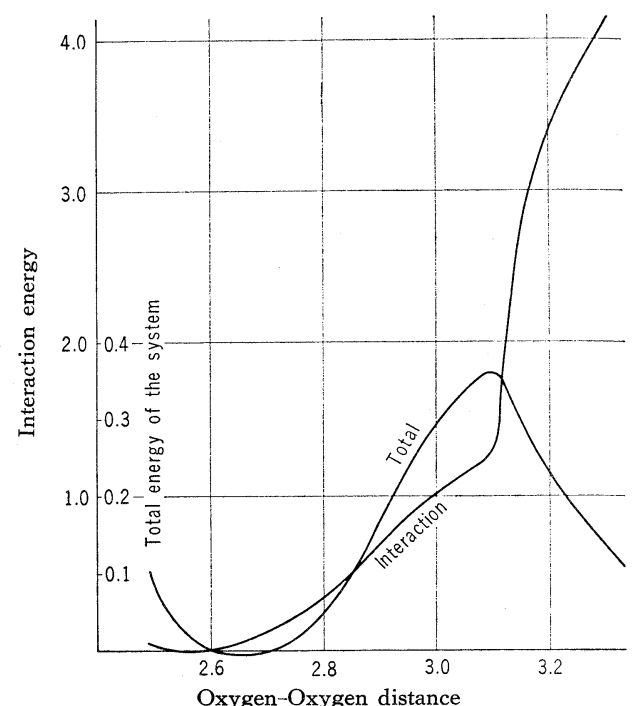

Fig. 4. Total energy and interaction energy between the nonbridging oxygens intermediated by the two sodium atoms. The values at 2.60 of the $\mathrm{O}-\mathrm{O}$ distance are taken as the base values.

ted as a functional of the distance. When Fig. 4 is drawn, the difference of the minimum point on the total energy curves between this system and the simplified system which has been constructed by neglecting the core orbitals to determine the positions of sodium atoms with the above mentioned meaning. That is about 0.01 short of the distance between the oxygens compared with the result achieved by use of the simplified model. Accordingly, we have to study the result admitting that some error is included in it.

The curve of valence orbital energy flattens out more and more at the shorter of the $\mathrm{O}-\mathrm{O}$ distances, resulting from deduction of the interaction of the oxygens with the sodiums because the sodium atoms move far from the oxygens on the line perpendicularly bisecting the middle point of the two oxygens with the decrease of the $\mathrm{O}-\mathrm{O}$ distance. The total energy decreases again above the distance 3.10 between the oxygens. In this region, referring to Table 2, the sodium atoms leave that line in order to take a configuration with the minimum energy of system, and each sodium atom changes its direction of movement toward one oxygen and the other respectively. That makes the interaction energy of each sodium atom with its nearest oxygen increase, and with the other oxygen decrease as shown in Fig. 3. Consequently, the decrease of energy being superior to the increase, the degree of total energy in the system drops. Part of this energy deduction comes from the core electron interaction which seems to be cause by the imperfect model because the core electron interaction is supposed not to decrease so much by being subjected to the effect of electron cloud 
in the discrete ion which is neglected from our consideration. Nevertheless, some difference in the interaction between the real and the imperfect model occurs both in the results of the finite separation and of the infinite between the oxygens. Thus we expect that the effect of electron cloud in the discrete ions becomes smaller in the difference of the results. Even if the effect of electron cloud is taken into consideration reasonably, there is a tendency to cause this situation in the glass system like seen in the valence electron interaction. It may be explained by the fact that the glass is said to be in a quasi-equilibrium state. That is : The constituent discrete molecules of the glass in a liquid state hold rather considerable energy if no account is taken of the interaction between the bridging oxygens and silicate atoms as seen in the result calculated in the infinite separation between the oxygens, but their interaction energy with each other is small. The former increases and the latter decreases as the temperature of the system drops. Since the result is that the variation of the former is superior to that of the latter, the total energy in the system rises if each discrete molecule adheres to the structure as the molecular interaction comes from the origin intermediated by the sodium atoms. A silicate-oxygen bond in the discrete molecule, however, is so strong that considerable entropy corresponding to rearrangement among the sodium, silicate and oxygen atoms is inevitable in the system, thus the rearrangement cannot occur in the temperature corresponding to the configuration considered over a short period of time. (If it occurs, the system follows a path to crystallization.) In consequence, the total energy in the system rises, still in unstable state, until the point $a_{2}$ when it begins to drop again, and hence the system changes into the eqilibrium state of the glass.

In Fig. 3, we show the energy calculated in the infinite separation between the oxygens. (here, the value of the abscissa means the relative positions between the sodium and oxygen at the indicated $\mathrm{O}-\mathrm{O}$ distance in the finite separation.)

The total energy decreases abruptly above 3.10 along the abscissa. Considering it with the result obtained in the finite separation between the two oxygens, one can see that the discrete molecule becomes stable individually above this point, and that the system begins to have a property as the simple assembly of the many isolated molecules.

That property is not solid but rather liquid, which means that the glass changes from the glass state to the supercooled one.

We take a difference defined as $V$ between the total energies of the finite and infinite separation between the oxygens, and regard this the interac- tion energy between the oxygens in the glass system. It is not the so-called bonding energy because no positions of the sodium atoms in the infinite separation between the oxygens coincide with the equilibrium configuration witn respect to the oxygen and sodium atom as shown in Fig. 3 so as to give rise to the minimum energy of interaction between them. Therefore the abrupt rise of the $V$ above the 3.10 along the abscissa shows that the interaction between the two nonbridging oxygens belonging to one discrete ion and another diminishes rapidly above this point. This point just coincides with the point at which the interaction of sodium atoms with the two nonbridging oxygens changes from the equal interaction with them into the different one with one and another of them, which has been denoted as the point $a_{2}$ in the preceding paper.

\section{Some remarks on the assumptions}

(A) Variation of the distance between the oxygens causes rearrangement of the sodium atoms in order to form a configuration giving rise to the minimum energy of the system at the resultant $\mathrm{O}-\mathrm{O}$ distance. Thus the interaction energy of the sodium atom with the oxygen, which act on each other even in the infinite separation between the oxygens, may be regarded as constituting a part of the effective potential energy between the oxygens in the four atoms' model. In the real system, however, locus of vibrational motion of the sodium atoms seems not to follow the path being accompanied with vibrational motion of the oxygens which is shown in Table 2 in order to make the system energy minimum. It is preferable to think that the sodium atoms remain in their mean positions determined by the temperature of the system in spite of the vibrational motions of the oxygens, and that they make the vibrational motions around these positions subjected to the considerable effect of other atoms, mainly, of course, of the nearest nonbridging oxygen. By this consideration, the positions of sodium atoms shown in Table 2 which are determined by making use of the simplified model should be regarded as their mean positions corresponding to the mean $\mathrm{O}-\mathrm{O}$ distance determined by the temperature of the glass system. It means that the motions of sodium atoms cannot be described precisely as the functional of the $\mathrm{O}-\mathrm{O}$ distance.

Moreover, when we discuss the relative motion of the two discrete molecules, variation of energy in the molecule, even if it is caused by the variation of the distance between the molecules, seems to have no relation to the molecular force between them which is obtained as derivative of the interaction energy. If this is not so, when the discrete 
molecules should interact with each other according to the potential shown as the total energy in Fig. 4, the glass system may be broken above the point 3.10. By these two considerations it is not unreasonable to introduce the assumption (D) of section 1 into the analysis of the model.

(B) Nevertheless, the deduction of effective potential energy above the point 3.10 is too abrupt compared with the effective potential assumed in the preceding paper. This derives from the assumption $(D)$ of section 1 , that is

$$
V^{\prime}(\mathrm{Na}-D)+V^{\prime}(\mathrm{Na}-\mathrm{O})=\text { constant }
$$

The interaction of the sodium atom is constituted mainly by its interaction with the nearest nonbridging oxygen, therefore the variation of it, $\delta V^{\prime}(\mathrm{Na}-\mathrm{O})$, caused by displacement of the sodium atom may not be cancelled out perfectly by variation of the interaction energy, $\delta V^{\prime}(\mathrm{Na}-D)$, between that sodium and atoms other than the nearest nonbridging oxygen. The resultant variation, $\delta\left(V^{\prime}(\mathrm{Na}-\mathrm{O})+V^{\prime}(\mathrm{Na}-D)\right)$, follows the variation of the first term. In the region above the point $a_{2}$, the $\delta V^{\prime}(\mathrm{Na}-\mathrm{O})$ drops so remarkably that it contributes to the effective potential so as not to rise as the curve of interaction energy shown in Fig. 4. This is the tendency we desire.

(C) We derive the effective potential $V$ between the oxygens from the total energies of the two models on the basis that one of them is formed from the finite separation between the oxygens and the other is from the infinite separation. The value of $V$ calculated is too low compared with the real interaction energy, even if it is taken into account that the configurations used to calculate the energy in the infinite separation are not the equilibrium one with respect to the interaction between the sodium and oxygen. We can easily find the origin of this poor value. That is; the interaction of the sodium atom with the oxygen in the four atoms' model cannot be replaced by that in the infinite separation between the oxygens. Furthermore, the interaction among the four atoms cannot be calculated separately in the system by reason of the quantum mechanical definition of the electron. Judging from the variation of total energy calculated by making use of this model in the infinite separation, however, we may be allowed to presume that the interaction of the sodium atom with the oxygen in the finite separation varies according to the manner resembling it. Therefore the characteristic form of the effective potential - that it rises abruptly at the point $a_{2}-$ is presumed to occur even if it were calculated.

(D) Generally the configuration of the sodium atoms and nonbridging oxygens does not form the group symmetry $C_{2 h}$, therefore the point $a_{2}$ is supposed not to appear so clearly as this result.

\section{Conclusion}

(A) The feature of effective potential intermediated by the two sodium atoms between the nonbridging oxygens is obtained and the characteristic point $a_{2}$ in it corresponding to the glass transition point is explained physically as a specific point of the interaction among them.

(B) The electronic state among them is made clear in both the finite and infinite separation between the nonbridging oxygens.

(C) The situation of transformation from the liquid to the glass state is discussed from the view point of the effective potential obtained.

\section{Acknowledgement}

One of us (Katsuki) would like to express his appreciation to Professor Huzio Nakano and the staff in his research group in Nagoya University, and also to Professor Sigeru Huzinaga in Kyusyu University who allowed him to use his program for gaussian expansion method for molecular integrals. We would also like to thank Mr. Takao Otozai, the manager of the Research Laboratory, for his kind encouragement.

Thanks are also due to Mr. Iga, Mr. Kondo and his colleague who have aided us in completing this work.

\section{References}

1) K.Katsuki, Yogyo-Kyokai-Shi 78 [6] 177-88 (1970).

2) C.C.J. Roothaan, Rev. Mod. Phys. 23, 69 (1951).

3) For instance, S. Yomosa, T. Miyata and H. Suzuki, J. Phys. Soc. Japan 24, 878 (1968).

4) S. Huzinaga, J. Phys. Soc. Japan 21, 2306, 2313 (1966).

5) R.S. Malliken, J. Chem. Phys. 23, 1833, 1841 (1955) ; Handbuch der Physik, Band XXXVII/2, Moleküle II, 80. [Received Mar. 10, 1970]

\section{$\mathrm{Na}_{2} \mathrm{O} \cdot 2 \mathrm{SiO}_{2}$ ガラスのガラス変態理論}

(非架橋酸素間の電子状態)

\section{香月一雄・青木厚二 \\ (日本板硝子 (株) 研究所)}

それぞれ異なった discrete ion に結合している2つの 非架橋酸素と，2 つのナトリウムよりなるモデルを用い て, ナトリウムに介存された非架橋酸素閒の相互作用を 簡単な SCF 理論を用いて求めた. その結果，4 原子系 内に作られる相互作用軌道の結合性㧍よび電子密度が求 められた，同様な手法を用いて，2つの非架橋酸素が無
限に離れた場合の状態を求めた. 求められた結果から前 報1)でガラス変態を生ずるに重要な役割をはたした非架 橋酸素間の有効ポテンシャルが求められた.このポテン シャルの観点よりガラスの準平衡状態について簡単に議 論した.

(3/10/1970 受付) 